\title{
Open Innovation in der Dienstleistungsgestaltung
}

\author{
Kathrin M. Möslein/Ralf Reichwald/Marcus Kölling
}

\begin{abstract}
Vielen gilt das Phänomen der Open Innovation, des offenen Innovierens zwischen verteilten Akteuren, als grundlegend neues Paradigma mit enormen Potenzialen für den Einzelnen, für Organisationen und ganze Volkswirtschaften. Andere sehen in der intensiven Beschäftigung mit Open Innovation bestenfalls einen Modetrend, wenn nicht gar ein Risiko für das geistige Eigentum des Innovators und die erfolgreiche Umsetzung der Innovation im Unternehmen. Der vorliegende Beitrag skizziert Potenziale und Spannungsfelder der Open Innovation für die Dienstleistungsgestaltung. ${ }^{1}$
\end{abstract}

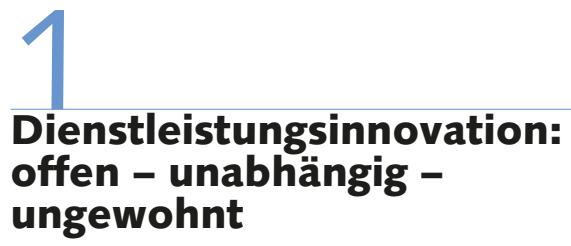

Open Innovation bezeichnet Innovationsprozesse, die nicht an den Grenzen von Unternehmen oder deren Innovationsabteilungen enden, sondern Akteure - unabhängig von deren institutioneller Zugehörigkeit - als Ideengeber, Konzeptentwickler oder auch Innovationsumsetzer in die Gestaltung von Innovationen einbinden (Möslein/Neyer 2009). Das Phänomen der Open Innovation geht dabei einher mit einem Perspektivenwechsel weg von einem Verständnis rein unternehmensdominierter Wertschöpfung hin zu einer Konzeption von Wertschöpfung und Innovation als interaktivem Geschehen (von Hippel 1978, 2005; Reichwald/Piller 2009). Derart interaktive Wertschöpfung und Innovation floriert in westlichen Industrienationen und Schwellenländern gleichermaßen, wenngleich mit unterschiedlichen Akzenten und Schwerpunktsetzungen. Wenn Dienstleistungsinnovation dergestalt offen, interaktiv und nutzergetrieben stattfindet, erscheint uns dies vielfach noch ungewohnt. Und doch ist das Phänomen längst allgegenwärtig. Zwei Beispiele mögen dies verdeutlichen. Die Beispiele sind ungewöhnlich, passen scheinbar nicht zu unserer Arbeits- und Unternehmenswelt und finden doch hier und jetzt statt. Sie sind Realität, zeigen wie gemeinsames Innovieren zu einem Element der Freizeitgestaltung wird und repräsentieren ein selbstverständlich nicht dominantes, jedoch vielerorts emergentes Phänomen mit hohem Begeisterungsfaktor für viele und zugleich Schreckenspotenzial für andere: Deutschland, an einem Sonntag im März 2011:

\subsection{BEISPIEL 1: \\ DER GLOBAL SERVICE JAM (WWW.GLOBALSERVICEJAM.COM)}

Es ist Sonntagnachmittag in Deutschland. Weltweit wird seit knapp 48 Stunden begeistert innoviert. Die Initiative „Global Service Jam“, von Markus Edgar Hormeß und Adam Lawrence in Nürnberg ins Leben gerufen, startete am Freitag zunächst in Neuseeland. Rund 60 Standorte weltweit, zuletzt San Francisco, erhielten jeweils zeitversetzt den Startschuss zur Mitwirkung. Sie hatten sich im Vorfeld als Standorte online registriert und lokal Freiwillige zur Mitwirkung eingeladen. Die Initiative hat einen klaren Zeitplan (48 Stunden von Freitagnachmittag bis Sonntagnachmittag), definierte Regeln im Spannungsfeld von Kooperation und Wettbewerb (kooperiert wird dezentral verteilt offline sowie auf allen erdenklichen Online-Kanälen, über den Themenfokus jedoch darf online nicht gesprochen werden) und ein festes Ziel (die Entwicklung innovativer Services zum Thema „(super) hero“). Die so weltweit in Gang gesetzte Innovationsaktivität zur Serviceentwicklung beeindruckt in Intensität und Begeisterung ebenso wie in Disziplin und Zielorientierung.

Das Geschehen lässt sich online u.a. auf www.globalservicejam.com, Twitter, Facebook, Vimeo, Youtube und UStream live mitverfolgen. Die Begeisterung ist ansteckend. Die Beteiligten haben offenkundig großen Spaß und verlieren doch ihr Ziel nicht aus den Augen. Denn im Ergebnis geht es darum, wer in diesem weltweiten Innovationswettbewerb fränkischer Herkunft letztlich als Sieger der Serviceentwicklung hervorgeht. Rund 190 Projekte sind am Start. Die Aufmerksamkeit ist groß, die Liste der Unterstützer hat durchaus Gewicht und umfasst Persönlichkeiten wie Joseph Pine, Autor des Bestsellers „Mass Customization“ aus den 1990er Jahren, oder Tony Hsieh, Gründer und Chief Executive Officer (CEO) von Zappos. Die Selbstorganisation wildfremder Teilnehmer, die sich standortübergreifend überhaupt nicht kennen und lokal vor 48 Stunden in der Regel zum ersten Mal aufeinandertrafen, ist überwältigend. Mit fortschreitender Zeit wird der Wettbewerb immer intensiver. Doch um 15:30 Uhr wird

\footnotetext{
1 Dieser Beitrag basiert auf Möslein/Neyer 2009 sowie Habicht et al. 2011. Grundlage bildet zum einen das Verbundforschungsprojekt "Open-I:

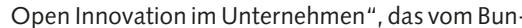
desministerium für Bildung und Forschung (BMBF) und dem Europäischen Sozialfonds (ESF) unter den Förderkennzeichen (FKZ) 01FM07053 (HHL), 01FM07054 (FAU) und 01FM07055 (TUM) gefördert wird (siehe auch: www.open-i.eu), zum anderen das Verbundforschungsprojekt "ServProf - Service-Professionalität lernen und leben“" (FKZ 01FB08043; siehe auch www.servprof.de).
}

Kathrin M. Möslein, Prof. Dr., ist Inhaberin des Lehrstuhls für Betriebswirtschaftslehre, insbesondere industrielle Informationssysteme, an der Universität ErlangenNürnberg und Mitglied des Direktoriums des Center for Leading Innovation \& Cooperation (CLIC) an der Handelshochschule Leipzig. Arbeitsschwerpunkte: Open Innovation, Hybride Wertschöpfung. e-mail: kathrin.moeslein@hhl.de Ralf Reichwald, Prof. Dr. Prof. h. c. Dr. h. c., ist Forschungsprofessor und Mitglied des Direktoriums des Center for Leading Innovation \& Cooperation (CLIC) an der Handelshochschule Leipzig und Emeritus of Excellence der Technischen Universität München. Arbeitsschwerpunkte: Interaktive Wertschöpfung, Dienstleistungsinnovation. e-mail: ralf.reichwald@hhl.de Marcus Kölling, Dr., ist Research Fellow am Center for Leading Innovation \& Cooperation (CLIC) an der Handelshochschule Leipzig. Arbeitsschwerpunkte: Dienstleistungsinnovation, Dienstleistungsprofessionalisierung. e-mail: marcus.koelling@hhl.de 
es plötzlich still im Web: es ist die „minute of silence for Japan" des Global Service Jam. Die scheinbar globale Spaßunternehmung der Dienstleistungsinnovation wirkt - nicht nur in dieser Minute - beeindruckend erwachsen, verantwortungsvoll und überaus organisiert. Am Ende haben 1.263 registrierte „Jammer" weltweit in 48 Stunden „übers Wochenende“ insgesamt 203 neue Servicedesigns erstellt.

\subsection{BEISPIEL 2: \\ UNSERALLER - WO PRODUKTENT- WICKLUNG ZUR SERVICE EXPERI- ENCE WIRD (WWW.UNSERALLER.DE)}

Zur gleichen Zeit läuft auch die Zeituhr bei unserAller unbarmherzig: nur noch 01 Tag, 01 Stunde, 10 Minuten und 10 Sekunden, dann geht das aktuelle Entwicklungsprojekt in die nächste Phase. Rund 5.660 registrierte Nutzer zählen aktuell zum freiwilligen Entwicklerkreis der unserAller-Facebook Community. Hier geht es scheinbar um klassische Produktentwicklung, um neue Senfsorten, um Chutney-Geschmacksrichtungen, das unserAller Traum- und Schaumbad „Badebombe“, Salatdressings und gerade eben aktuell um die Entwicklung eines neuen Balea-Duschbads für die kalte Jahreszeit: „Ziel dieses Projektes ist die Kreation eines Konzeptes für eine neue Dusche, die Balea im Herbst 2011 einführen und in Eurem dm-Markt erhältlich sein wird. Ihr seid aufgefordert, in 4 Phasen Eigenschaften für diese neue Dusche einzureichen" (www. unseraller.de). Der mehrphasige Entwicklungsprozess ist straff organisiert, an seinem Ende steht regelmäßig termingerecht ein einfaches neues Produkt, das schon vor seiner Markteinführung über hunderte freiwilliger „Markenbotschafter“ verfügt, die die Entwicklung online und offline getragen haben. Es wird online diskutiert, konfiguriert, beraten und bewertet. Ausgewählte Teilnehmer erhalten sog. "Selbermachpäckchen" nach Hause. Dort wird dann experimentiert, ausprobiert, Erfahrung gesammelt, fotografiert und zurückgespiegelt in die Online-Community. Im Prozess der Duschbadentwicklung ist die Entscheidung über das Produktmotto bereits gefallen. In nur einer Woche wurden 1.181 Vorschläge für das Motto des Duschbads eingereicht. Durchgesetzt hat sich das Motto „Diamonds \& Ice“. Doch welche Produkteigenschaften würden ein derartiges Duschbad auszeichnen? Kon- figuriert wird in den Dimensionen Farbe, Konsistenz, Schimmer sowie Geruch online mit dem Misch-Konfigurator im Rahmen der Facebook-Applikation ebenso wie offline auf Basis der Balea-Ausprobierund Selbermachpäckchen, welche die 750 aktivsten Mitentwickler bereits im Vorfeld der Konfigurationsphase per Post erhalten haben. Die Vision der vier jungen Münchner unserAller-Gründer ist klar: „Bei unserAller entscheidest Du, wie neue Produkte aussehen!“. Die Unternehmensbeschreibung liest sich ungewöhnlich: „Eure Ideen sind Gold wert! Viele Firmen vergessen das gerne und produzieren oft, was die Menschheit nicht braucht. Und genau deswegen nimmt sich unserAller Euren Vorschlägen an. Entwickelt gemeinsam Produkte und bestimmt mit, wie sie aussehen, was sie können und wie sie heißen. Am Ende werden Eure Vorschläge genau so umgesetzt, wie Ihr das wollt und Ihr könnt stolz sein auf Euer - nein - unserAller Produkt!“ (ebd.). Was hier als Produktunternehmen mit Fokus auf Produktentwicklung auftritt, ist eine Plattform für offene interaktive Innovation, für Service Experience und Community-Organisation. UnserAller arbeitet an selbstinitiierten ebenso wie unternehmensbeauftragten Produktentwicklungsprozessen und organisiert diese als interaktiven Community-Prozess. Das Produkt wird somit unmittelbar zur hybriden Leistung, der Prozess seiner Entwicklung zum interaktiven Wertschöpfungsprozess der Open (Service) Innovation.

Wie die oben skizzierten Fallbeispiele zeigen, bewegen wir uns in einer neuen Ökonomie jenseits der in der Institutionenökonomie bekannten Markt- und Unternehmensformen (Picot et al. 2003). Im beschriebenen Prozess der Entwicklung und Gestaltung von Dienstleistungen entsteht Wertschöpfung in neuen Arbeitsformen, die den bekannten Arrangements der betrieblichen und marktlichen Arbeitsteilung nicht zugeordnet werden können. Viele Fragen sind offen: Fragen der Motivation, der Eigentumsrechte, des opportunistischen Verhaltens und der Vertrauensbildung. Die Regelung von Eigentumsrechten wird in der Open-Source-Debatte intensiv diskutiert (vgl. z. B. Henkel 2006), während die Open-Innovation-Diskussion in der sozialwissenschaftlichen Literatur vor allem Fragen der Motivation und der Vertrauensbildung aufgreift (vgl. z. B. Schattke/Kehr 2009).

\section{Perspektiven der Open (Service) Innovation}

Das Phänomen des offenen Innovierens ist keineswegs unstrittig. So beschreibt beispielsweise Henry Chesbrough von der University of California (UC) Berkeley in seinem einflussreichen Buch „Open Innovation" diese Form der Innovation als neuen Imperativ der Wertschöpfung und kontrastiert das Modell klar mit dem Gegenpol einer geschlossenen Innovation (Chesbrough 2003). Andere Autoren sehen in der intensiven Beschäftigung mit Open Innovation bestenfalls einen Modetrend, wenn nicht gar ein Risiko für das geistige Eigentum des Innovators und die erfolgreiche Umsetzung der Innovation in Wettbewerbsvorteile für das innovierende Unternehmen.

Was also verbirgt sich hinter dem Konzept der Open Innovation und dem vielfach proklamierten Paradigmenwechsel? Was ist neu? Was ist relevant? Wo liegen die Herausforderungen und Chancen für Unternehmen? Im Folgenden gehen wir diesen Fragen auf die Spur. Zunächst werden Grundlagen und Hintergründe der Open Innovation skizziert. Dabei werden zunächst die für Unternehmen relevanten Grundparadigmen der Innovationsgestaltung herausgearbeitet und zur Diskussion gestellt (Abschnitt 2), um sodann wichtige Werkzeuge in Prozessen offener Innovation vorzustellen (Abschnitt 3). Im Anschluss daran wird auf Spannungsfelder einer erfolgreichen Realisierung insbesondere für die Dienstleistungswirtschaft verwiesen (Abschnitt 4).

Insgesamt herrschen in der Literatur heute zwei grundlegende Begriffsverständnisse vor, die sich jedoch lediglich in der gewählten Perspektive unterscheiden, in der Sache jedoch ergänzen; praktische Realisierungen weisen häufig Elemente beider Perspektiven auf. So lässt sich Open Innovation einerseits in Anlehnung an das Open Source-Verständnis der Welt der Softwareentwickler als ein Phänomen der weitgehend selbstorganisierten und eigenmotivierten, internetgestützten, verteilten, kollaborativen Entwicklung und Gestaltung von Innovationen verstehen. Diese Sichtweise startet beim unabhängigen Einzelakteur und stellt das interaktive Zusammenwirken solcher Akteure ins Zentrum. 
Sie verzichtet in ihrer Extremform auf die Institution der Unternehmung. Eric von Hippel, Professor am Massachusetts Institute of Technology (MIT) in Cambridge, geht daher in visionären Überlegungen soweit, zu postulieren, dass die Institution der Unternehmung und die Funktion des Managements für die Gestaltung von Innovationen insgesamt obsolet werden könnten (von Hippel 2005). Doch zeigt er damit nur einen möglichen Extrempunkt einer Entwicklung auf. Für das aktuelle und zukünftige Innovationsgeschehen in Unternehmen und Märkten ist diese Vision zwar inspirierend, aber dennoch etwas weit gegriffen. So beobachten wir heute, dass selbst aus dezentral verteilten Innovationsaktivitäten vielfach neue Unternehmen hervorgehen oder etablierte Unternehmen diese nutzen und im Extremfall gar vollständig integrieren. Die komplette Auflösung der Unternehmung und ihrer Innovationsfunktion steht damit nicht unmittelbar bevor. Dennoch befördert das Innovationsgeschehen der Open Innovation die Auflösung starrer Unternehmensgrenzen im Inneren wie auch am äußeren Rand der Unternehmung.

Dies führt uns zur zweiten Grundperspektive der Open Innovation, wie sie beispielsweise Henry Chesbrough von der University of California (UC) Berkeley beschreibt: Open Innovation bildet für ihn den Gegenpol zur klassischen Etablierung weitgehend hermetisch abgeschotteter F\&E-Labore (Forschung \& Entwicklung), der sogenannten „Closed Innovation“. So haben Unternehmen lange Zeit ihr Innovationsgeschehen weitestgehend im Bereich der F\&E gebündelt, dort hochrangige Experten beschäftigt, diese mit anspruchsvollen Innovationsaufgaben betraut, das geistige Eigentum in höchstem Maße geschützt und zugleich nur wenig am externen Innovationsgeschehen partizipiert. Mit der Industrialisierung und der Professionalisierung betrieblicher Funktionalbereiche (vgl. auch Baethge 2011) hat sich zunehmend ein internes Expertentum, eine grundsätzliche Präferenz interner Lösungen, ein ausgeprägtes „Not-invented-here“-Verhalten gegenüber externen Impulsen sowie eine deutliche Abschottung, insbesondere im Bereich der Forschung und Entwicklung, herausgebildet und wechselseitig verstärkt. Mit der Möglichkeit, in immer weiteren Bereichen Ideen, Konzepte und Innovationen mediengestützt, global verteilt, kollektiv über das Internet zu suchen und zu finden, $\mathrm{zu}$ entwickeln und auszutauschen, zu bewerten und zu selektieren sowie letztlich sogar umzusetzen und zu vermarkten, wird dieses klassische Modell zunehmend herausgefordert.

In den meisten Branchen hängt die Wettbewerbssituation langfristig im Wesentlichen von der Innovationsfähigkeit der Unternehmen ab. Innovationsfähigkeit wird dabei maßgeblich geprägt durch die organisatorischen Rahmenbedingungen für den Innovationsprozess, durch Innovationsklima und Innovationskultur, vor allem aber durch das Wissen und die Kompetenzen der Beschäftigten. Der Open Innovation-Ansatz versteht sich deshalb nicht nur als Konzept der Einbeziehung externen Wissens in den betrieblichen Innovationsprozess, sondern es geht gleichermaßen um das systematische Einbeziehen des Wissens der Mitarbeiter (vgl. z. B. Scholl 2006). Fragen der Partizipation und Interaktion der Beschäftigten des Unternehmens stehen damit im Zentrum des Innovationsprozesses und die Frage, welche Rollen Betriebsrat und Arbeitnehmervertretungen im Innovationsprozess des Unternehmens einnehmen können. ${ }^{2}$

Wenngleich eine derartige Öffnung des Innovationsgeschehens bisher primär von Unternehmen im Bereich der IT-Services praktiziert wurde, werden Ansätze der „Innovation und Mitbestimmung“"zunehmend auch in Unternehmen klassischer Industrie- und Dienstleistungsbranchen aufgegriffen. Bereits heute nutzen zahlreiche Dienstleister im öffentlichen wie privatwirtschaftlichen Bereich die Potenziale der Open Innovation. Insbesondere im Bereich öffentlicher Dienstleistungen versprechen derartige Prozesse der Open Innovation auch Alternativen zu Privatisierungsansätzen. Unter dem Stichwort „Open Government“ wird darüber hinaus aktuell an einer Systematisierung der Potenziale der Open Innovation für den Bereich der öffentlichen Verwaltung gearbeitet (vgl. z. B. Hilgers et al. 2010).

Dienstleistungsunternehmen, die den Open-Innovation-Ansatz nicht nur für Externe, sondern auch für die eigenen Mitarbeiter in der Breite des Unternehmens öffnen wollen, brauchen geeignete aufbau- und ablauforganisatorische Regeln, ein offenes Innovationsklima und eine offene Innovationskultur, in welcher der Wissenstransfer ohne Barrieren und Misstrauen stattfinden kann. Dies alles verlangt neue Kompetenzen bei allen Beteiligten. Für offene Innovationsprozesse benötigen die Akteure insbesondere sogenannte Interaktionskompetenzen (vgl. Reichwald/ Piller 2009). Bezüglich der Debatte um die organisatorischen und kulturellen Voraussetzungen für das Innovationsmanagement im Unternehmen muss auf die aktuelle sozialwissenschaftliche Debatte verwiesen werden (vgl. z. B. Zerfass/Möslein 2009; Baethge 2011). Im Folgenden soll speziell auf ein ergänzendes Feld der Kompetenzentwicklung eingegangen werden, das hohe Potenziale für den Aufbau von Interaktionskompetenz für alle Akteure im Open-Innovation-Prozess enthält: die Werkzeuge der Open Innovation.

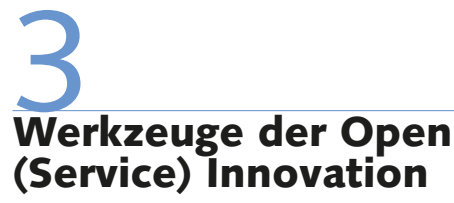

Beide oben skizzierten Grundperspektiven der Open Innovation sind im heutigen globalen Innovationsgeschehen Realität: Open Innovation als Phänomen der weitgehend selbstorganisierten und eigenmotivierten, internetgestützten, verteilten, kollaborativen Entwicklung und Gestaltung von Innovationen einerseits und Open Innovation als unternehmensinitiierte und organisierte Form der mediengestützten, verteilten und kollaborativen Innovationsgestaltung gemeinsam über die formalen Grenzen von Unternehmen hinweg andererseits. Beide Grundperspektiven werden in der praktischen Anwendung von IT-basierten Werkzeugen unterstützt. Der Blick richtet sich hier auf die fünf in Abbildung 1 dargestellten zentralen Werkzeugklassen, die heute den Trend zur Open Innovation unterstützen und vorantreiben. Ihre Entstehung, Fortentwicklung und Verbreitung gründet sich zentral auf Elemente von Social Software im Web 2.0. Alle fünf Werkzeugklassen sollen im Folgenden knapp vorgestellt und anhand von kurzen Beispielen erläutert werden.

(1) Innovationswettbewerbe rufen zur wettbewerblichen Lösung von konkreten Innovationsproblemen auf und schreiben hierfür Preise aus (vgl. Haller et al. 2011).

\footnotetext{
$2 \mathrm{Vgl}$. hierzu insbesondere das Schwerpunktheft der WSI-Mitteilungen (2010) "Innovation und Mitbestimmung " 63 (2)
} 
Historisch gesehen sind solche Wettbewerbe nicht neu: So schrieb das British Empire im Jahr 1714 den sogenannten „Longitude Prize“ für die Suche nach einem innovativen Verfahren der korrekten Berechnung von Längengraden aus. Das ausgeschriebene Preisgeld variierte in Abhängigkeit von der Präzision der Lösung. Doch schon im Jahr 1567 schrieb Philipp II, König von Spanien, für die gleiche Problemstellung einen „Innovationswettbewerb" aus. Auch Napoleon nutzte das Verfahren des Innovationswettbewerbs auf der Suche nach einem innovativen, insbesondere haltbaren und preiswerten Ersatzprodukt für Butter und bescherte der Welt so die Margarine. Was also ist neu und was macht Innovationswettbewerbe heute zu so einem zentralen Werkzeug der Open Innovation? Die Antwort ist einfach: Heute eröffnet das Internet letztlich jedem Einzelnen die wettbewerbliche Ausschreibung von spannenden Innovationsfragestellungen - global sichtbar zu vergleichsweise geringen Kosten. Um damit Erfolge zu erzielen, muss man noch immer genügend Aufmerksamkeit garantieren, sodass vielversprechende Innovatoren auf den Wettbewerb aufmerksam werden und tatsächlich teilnehmen. Hierzu bieten die Mechanismen des Web 2.0 für Unternehmen die besten Voraussetzungen. Neu ist damit die Möglichkeit, die Methode des Innovationswettbewerbs für Unternehmen als Innovationswerkzeug gerade auch für die Dienstleistungsentwicklung zu nutzen. Die Auslotung weiterreichender Möglichkeiten der Nutzung von Innovationswettbewerben zur Adressierung gesellschaftlicher Herausforderungen ist derzeit ein Forschungs- und Gestaltungsfeld von höchster Relevanz. Spannende erste Ansätze zeigen sich im Einsatz von Innovationswettbewerben zur Steigerung der Energieeffizienz von Städten (www. saveourenergy.de), zur Erarbeitung neuer Versorgungslösungen für seltene Erkrankungen (www.gemeinsamselten.de) oder zur radikalen Qualitätssteigerung von Lehrangeboten in Massenstudiengängen (vgl. hierzu und zu zahlreichen weiteren Praxisbeispielen das Innovation Contest Inventory, www.innovation-contest.de).

(2) Innovationsmarktplätze sind virtuelle Orte, an denen Angebot und Nachfrage zusammentreffen. Sie werden typischerweise über webbasierte Plattformen realisiert, auf denen einerseits Innovationssucher (ty- pischerweise Unternehmen) Innovationsprobleme ausschreiben und andererseits Innovationslösungsanbieter (typischerweise Einzelinnovatoren oder Innovatorenteams) konkrete Lösungskonzepte für diese Probleme vorschlagen. Sie vermitteln damit als Intermediäre zwischen Innovationsanbietern und -nachfragern. Im Internet stehen für Unternehmen hunderte solcher Intermediärsplattformen für die Innovationsausschreibung bereit. Einige können heute bereits als etabliert angesehen werden, sie werden von Unternehmen auch bereits im großen Stil genutzt. Einer der bekanntesten und etabliertesten Innovationsmarktplätze ist Innocentive. Innocentive wurde bereits im Jahr 2001 von Eli Lilly gegründet und bezeichnet sich selbst als "the world's first Open Innovation Marketplace“ (www. innocentive.com). Dieser Innovationsmarktplatz startete ursprünglich mit einem klaren Fokus auf Innovationsherausforderungen der chemischen Industrie. Heute bedient Innocentive Unternehmen in über 40 Industrien und 200 Ländern weltweit. Über 200.000 Innovatoren sind derzeit Mitglied in der Innocentive-Community der Lösungsanbieter. Unternehmen bieten ihnen Innovationsausschreibungen mit einem Preisgeld von rund 5000 \$ bis zu 1.000.000 \$. Als Intermediär bündelt Innocentive damit Innovationswettbewerbe über die Grenzen von Unternehmen, Branchen und Märkten hinweg. Innovationsmarktplätze wie Innocentive haben heute nach rund zehn Jahren der Etablierung ihren Fokus längst auf die Innovation von Services, Systems und Solutions gerichtet. Gesuche für Produktinnovationen stellen hingegen nur einen verschwindenden Teil der Marktplatzaktivitäten dar.

(3) Innovations-Communities ermöglichen Innovatoren, mit Gleichgesinnten gemeinschaftlich Ideen zu entwickeln, zu diskutieren und Innovationen voranzutreiben. Internetbasierte Innovations-Communities bündeln dabei typischerweise interessierte und kundige Innovatoren weltweit zu bestimmten Themen. So unterstützen sie als Innovationswerkzeug die gemeinsame Entwicklung und Fortentwicklung von Innovationskonzepten. Ihren Ursprung haben Innovations-Communities in freiwilligen und unternehmensunabhängigen Zusammenschlüssen Gleichgesinnter zur gemeinsamen Lösungsentwicklung. Die Community der Open-Source-Entwickler bildet ein typisches und überaus erfolg-

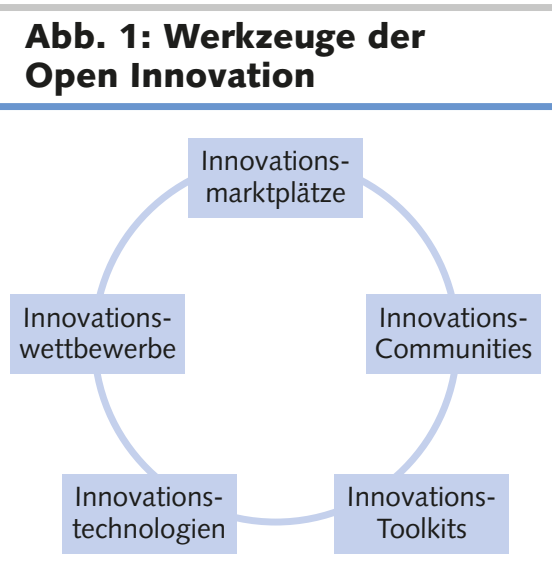

Quelle: Darstellung der Autoren.

WSI MITTEILUNGEN

reiches Beispiel. Doch haben längst auch Unternehmen Innovations-Communities als Werkzeug der Innovationsentwicklung für sich zu nutzen gelernt. Unternehmensgesteuerte Innovations-Communities als Instrument der Innovationsentwicklung treten daher heute mehr und mehr an die Stelle rein selbstorganisierter InnovationsCommunities. Ein besonders beeindruckendes Beispiel einer solchen InnovationsCommunity bildet die Apple Developer Connection (developer.apple.com). Diese internetbasierte Community lädt interessierte Innovatoren dazu ein, neue Anwendungen, Angebote und Lösungen rund um Apple-Produkte zu entwickeln. Dabei ist die Mitwirkung in der InnovationsCommunity für begeisterte Apple-externe Innovatoren mitnichten kostenfrei. Es existieren vielmehr unterschiedliche Formen und Intensitäten der Community-Mitgliedschaft, die mit unterschiedlichen Mitwirkungsrechten und Mitgliedsbeiträgen verbunden ist, wobei der Jahresbeitrag bis zu 3.500 \$ in der Premium-Mitgliedschaft reicht. Apple's Innovations-Community ist damit nicht länger nur Werkzeug der Innovationsentwicklung, sondern wird zugleich als Geschäftsmodell betrieben. Die Gestaltung von Betreibermodellen für Innovations-Communities wird damit für Unternehmen zu einer erfolgskritischen Herausforderung.

(4) Innovations-Toolkits bieten eine Entwicklungsumgebung, in der Nutzer Schritt für Schritt konkrete Innovationslösungen entwickeln können. Als internetbasiertes Innovationswerkzeug eröffnet es die Möglichkeit, eine große Zahl an Externen auch ohne spezifische Vorbildung in konkrete 


\section{Abb. 2: Effekte internetbasierter Werkzeuge der Open Innovation}

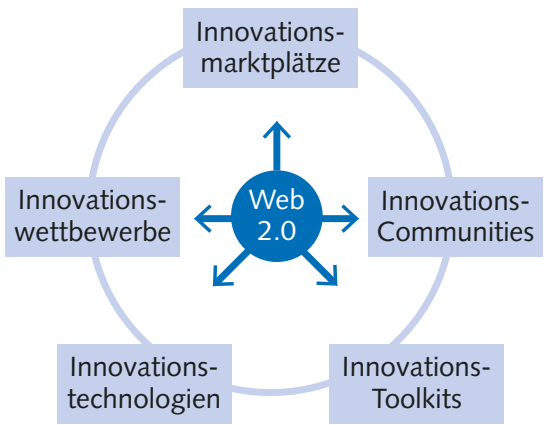

Quelle: Darstellung der Autoren.
Großzahlige Mitwirkung

Großräumige Verteiltheit Interaktion

Globales Gedächtnis
Geschwindigkeit der und strukturierte Innovationsprozesse einzubinden. Sie geben einen beschränkten Lösungsraum vor und stimulieren häufig gerade dadurch die Kreativität der Nutzer. Reichwald/Piller (2009) unterscheiden drei Grundtypen von Toolkits, die sich in ihrer Zielsetzung, den Gestaltungsprinzipien und der jeweiligen Nutzergruppe unterscheiden: Toolkits für User Innovation, Toolkits für User Co-Design sowie Toolkits zum reinen Ideentransfer. Unabhängig von der spezifischen Ausgestaltung lebt Toolkit-gestütztes Innovieren vom Feedback (sei es durch das System oder durch andere Nutzer), von der Simulation möglicher Lösungen (beispielsweise hinsichtlich Design, Leistung und Preis) und einer prozessorientierten Unterstützung der Lösungssuche. Die zentrale Herausforderung bei der Gestaltung liegt in der Definition der Freiheitsgrade des angebotenen Lösungsraums.

(5) Innovationstechnologien eröffnen schließlich den Schritt vom Design einer (Produkt-) Innovation zu ihrer Umsetzung und wandeln so den Prozess der Produktentwicklung zu dem einer Service Experience. Dabei sollen hier unter dem Begriff der Innovationstechnologien primär solche gefasst werden, die im Innovationsprozess die Schritte des Prototyping und der Umsetzung unterstützen und im Prinzip von jedermann über das Internet angesteuert werden können. Hierzu zählt beispielsweise die internetbasierte Ansteuerung von 3D-Scannern, 3D-Druckern oder Laserschneidegeräten. Mit einer Nutzerinteraktion mit derartigen Technologien wird die Erwartung einer weiteren radikalen Demokratisierung des Innovationsgeschehens und der vielfach proklamierte Trend hin zur „Personal
Fabrication" verbunden. In der Tat ist die Vision in Teilen heute bereits Realität: So bietet das Internet-Unternehmen Ponoko (www.ponoko.com) jedem Einzelnen die Möglichkeit, vielfältige Designs zu entwerfen, unmittelbar zu produzieren und zu vertreiben. Über Ponoko kann im Prinzip jeder Einzelne beispielsweise zu IKEA in Konkurrenz treten und innovative Produktangebote für sich selbst, aber eben auch für den Weltmarkt entwerfen, produzieren und vermarkten. Als Technologien des offenen Innovierens stehen diese Innovationstechnologien heute noch ganz am Anfang. Ihr wirklich nachhaltig sinnvoller Anwendungs- und Einsatzbereich wird sich erst noch herauskristallisieren. Es ist spannend zu beobachten, wie die wirkliche Open Product Innovation und das Open Manufacturing hier schon allein aus Gründen der noch unzureichenden Technologieentwicklung der Open Service Innovation weit nachstehen.

\section{4 Auf dem Weg zur Open Service Innovation: Implikationen und Schlussfolgerungen}

Die dargestellten internetbasierten Werkzeuge der Open Innovation weisen einige zentrale Effekte auf:

- Sie ermöglichen die großzahlige Mitwirkung von Akteuren am Innovationsprozess;

- sie erlauben die Zusammenarbeit dieser Akteure in großräumiger Verteiltheit;

- sie bewirken eine deutliche Erhöhung der Geschwindigkeit der Interaktion;
- sie stellen für die Akteure im Innovationsgeschehen ein globales Gedächtnis bereit.

Die vier genannten Effekte (Abbildung 2) erleichtern die Zusammenarbeit innerhalb und außerhalb von Unternehmen deutlich und eröffnen für den Einzelnen völlig neue Mitwirkungsmöglichkeiten an Innovationsprozessen. Man spricht in diesem Kontext daher auch von einem klaren Trend zur „Mitmach-Innovation“, der durch die Herausbildung und Verbreitung des Web 2.0 - des sogenannten „Mitmach-Webs“als Plattform für Innovationsaktivitäten vorangetrieben wird.

Die eingangs skizzierten Beispiele des Global Service Jam einerseits und der Innovationsentwicklung in der unserAller-Community andererseits nutzen eben diese Mitmacheffekte. Für Unternehmen eröffnen die genannten Effekte ebenfalls spannende Chancen. Ihre Realisierung bedingt jedoch vielfältige Herausforderungen. Die Innovationsforschung richtet dabei aktuell ihren Untersuchungsfokus primär auf die klassischen Spannungsfelder der Innovationsentwicklung, welche durch Entwicklungen der Open Innovation tendenziell verschärft werden. Diese sollen hier im Folgenden knapp skizziert werden.

(1) Einzelinnovator oder Innovationsteam: Das klassische Spannungsfeld zwischen Individualleistung und Teamleistung im Innovationsprozess gewinnt durch Open Innovation weitere Brisanz. Denn global verteilt werden weitere Akteure - Einzelne wie Teams - in das unternehmerische Innovationsgeschehen eingebunden. Darüber hinaus aber erhält die Gemeinschaft der Innovatoren, die Innovations-Community, als zunehmend internetbasierte Innovationsgemeinschaft in der Open Innovation sukzessiv einen dominanten Status. Eine lose Kopplung von Einzelnen und Teams über die Grenzen von Organisationseinheiten und Organisationen hinweg etabliert sich allmählich als Standardform der Zusammenarbeit im Innovationsgeschehen. Die Frage der Zurechnung der Innovationsleistung zum Innovator gewinnt in derart lose gekoppelten Strukturen gerade bei Prozessen interaktiver immaterieller Innovation, wie sie im Bereich der Dienstleistungsinnovation vorherrscht, neue Brisanz. Andererseits unterstützt die internetbasierte interaktive Innovation auch 
eine Dokumentation von Einzelbeiträgen auf Mikroebene (wie dies beispielsweise aus Wikipedia oder der Entwicklung von Open Source Software weithin bekannt ist). Unabhängig davon aber kommt die Möglichkeit der IT-basierten offenen Einbindung verteilter Akteure der Forderung nach einer wirklich aktiven Einbindung von Kunden in die Prozesse der Serviceinnovation unmittelbar entgegen.

(2) Lokale Innovation oder globale Verteilung: Lange galt die Frage nach einer lokalen Bündelung versus globaler Verteilung der Innovationsaktivitäten als eine Frage des „Entweder-Oder“. Doch erfolgreiche Realisierungen der Open Innovation machen mehr und mehr deutlich, dass heute die Voraussetzungen gegeben sind, um globale Kreativität und Innovationskraft von vielen verteilten Akteuren lokal zu bündeln und mit lokalen Stärken gezielt und komplementär zu kombinieren (vgl. auch Böhle et al. 2008). Die Werkzeuge der Open Innovation ermöglichen diesen Schritt. Das Beispiel des Global Service Jam zeigt eindrucksvoll, wie auch selbstorganisierte lokale Teams selbst kurzfristig in global verteilte Innovationsprozesse eingebunden werden können. Lokale Kompetenzen bei global verteilter Innovation in Unternehmen zielorientiert und wertschöpfend zu nutzen, bleibt jedoch eine zentrale Herausforderung für das Management von Open Innovation.

(3) Innovationsschritt oder Sprunginnovation: Während lange Zeit die Überzeugung dominierte, Unternehmensexternekönnten nur zu inkrementellen Innovationen Beiträge leisten, zeigen zahlreiche Erfolgsbeispiele heute, dass Open Innovation sowohl für kontinuierliche wie auch diskontinuierliche Innovationen - für Innovationsschritte und Innovationssprünge - seine Berechtigung und Unterstützungspotenziale aufweist. Die Herausforderung liegt in der konkreten Ausgestaltung, Umsetzung und strategischen Ausrichtung im Unternehmen. Nicht zuletzt die Entwicklung von Apples iPod im Verbund mit der Serviceinfrastruktur iTunes ist ein treffendes Beispiel für die Realisierung einer Sprunginnovation, die individuelles Verhalten genauso verändert hat wie die Grenzen klassischer Marktsegmente oder die ökonomischen Spielregeln der betroffenen Branchen. Während die oben aufgezeigten Beispielfälle im Ergebnis jeweils eher inkrementelles Innovieren erkennen lassen, bilden sie in ihrer organisatorischen Anlage jedoch bereits eine Sprunginnovation. Spannend zeigt sich hierbei, wie sich in interaktiver Innovation die Rollen der Akteure (vom Kunden zum Innovator und Unternehmer) ebenso verschieben wie die Grenzen von Produkt und Dienstleistung zu letztlich interaktiven hybriden Leistungsbündeln (Möslein/Kölling 2007; Spath/Ganz 2009).

(4) Geschlossen oder offen Innovieren: Nicht zuletzt stellt die Möglichkeit der Open Innovation Unternehmen vor die Herausforderung, das Spannungsfeld zwischen geschlossenem und offenem Innovieren zu meistern, denn es geht nicht darum, eine einseitige Öffnung zu betreiben oder "Closed Innovation" komplett durch „Open Innovation“ abzulösen. Vielmehr sind balancierte Strategien notwendig, die die Vorteile der Öffnung mit den Stärken der Geschlossenheit kombinieren, ohne dabei die Nachteile beider Spielarten der Innovation zu kombinieren.

Chesbrough macht in seinem neuen Buch „Open Services Innovation“ (2011) die Zielfunktion deutlich. Er betont den „Commodity Trap“ - also die Falle der undifferenzierten Leistungsangebote - als zentrale Herausforderung für Unternehmen im globalen Wettbewerb. Die offene Integration der intendierten Abnehmer als zentrale Akteure im Leistungsentwicklungs-, Leistungserstellungs- bzw. -erbringungsprozess zu gestalten, verspricht Auswege aus dieser Falle. Open Innovation in der Dienstleistungsgestaltung bildet vor diesem Hintergrund aus betriebswirtschaftlicher Perspektive eine zentrale Differenzierungsoption mit weitreichenden, zumeist noch ungenutzten Potenzialen. Doch zeigt sich auch, dass die von der betriebswirtschaftlichen Innovationsforschung derzeit in den Blick genommenen Spannungsfelder bei Weitem nicht die einzigen sind, die beim Einsatz von Open Innovation in der Dienstleistungsentwicklung zu bewältigen sind. Sozialwissenschaftliche Disziplinen seien daher in der Breite aufgerufen, sich mit den spannenden Potenzialen, aber gerade auch mit den Spannungsfeldern ihrer Realisierung im weiteren Sinne auseinanderzusetzen.

\section{LITERATUR}

Baethge, M. (2011): Kompetenzentwicklung und Professionalisierung im Dienstleistungssektor - Einige Diskussionsanregungen, in: Reichwald, R./Möslein, K. M./Kölling, M. (Hrsg.): Professionalisierung im Dienstleistungsbereich, CLIC Executive Briefing, Note (018), Leipzig, S. 17-21 Bessant, J./Davies, A. (2007): Managing service innovation, in: DTI Occasional Paper (9), Innovation in Services. Department for Trade and Industry, London, S. 61-95

Böhle, F./Bolte, A./Pfeiffer, S./Porschen, S. (2008): Kooperation und Kommunikation in dezentralen Organisationen, in: Funken, Ch./SchulzSchaeffer, I. (Hrsg.): Die Digitalisierung der Arbeitswelt, Wiesbaden, S. $93-115$
Chesbrough, H. W. (2003): Open Innovation: The New Imperative for Creating and Profiting from Technology, Boston

Chesbrough, H. W. (2011): Open Service Innovation, Rethinking Your Business to Grow and Compete in a New Era, San Francisco

Habicht, H./Möslein K. M./Reichwald, R. (2011): Open Innovation: Grundlagen, Werkzeuge, Kompetenzentwicklung, in: IM Information Management und Consulting 26 (1), S. 44-51

Haller, J./Bullinger, A. C./Möslein, K. M. (2011): Innovationswettbewerbe - Ein IT-gestütztes Instrument des Innovationsmanagements, in: Wirtschaftsinformatik (im Erscheinen)

Henkel, J. (2006): Selective revealing in open innovation processes: The case of embedded Linux, in: Research Policy 35 (7), S. 953-969 
Hilgers, D./Ihl, C./Vossen, A./Piller, F. (2010): The Era of Open Government - Harnessing the Benefits of Openness for Public Innovation. Paper presented at the 10th European Academy of Management (EURAM)

Conference 2010

Möslein, K. M./Kölling, M. (2007): Interaktive hybride Wertschöpfung als Innovationsstrategie, in: Streich, D./Wahl, D. (Hrsg.): Innovationsfähigkeit in einer modernen Arbeitswelt, Frankfurt, S. 195-202

Möslein, K. M./Neyer, A.-K. (2009): Open Innovation: Grundlagen, Grenzen, Spannungsfelder, in: Zerfaß, A./Möslein, K. M. (Hrsg.): Kommunikation als Erfolgsfaktor im Innovationsmanagement - Strategien im Zeitalter der Open Innovation, Wiesbaden, S. 85-103

Picot, A./Reichwald, R./Wigand, R. (2003): Die Grenzenlose Unternehmung, Wiesbaden
Reichwald, R./Piller, F. T. (2009): Interaktive Wertschöpfung - Open Innovation, Individualisierung und neue Formen der Arbeitsteilung, Wiesbaden

Schattke, K./Kehr, H. (2009): Motivation zur Open Innovation, in: Zerfass, A./Möslein, K. (Hrsg.): Kommunikation als Erfolgsfaktor im Innovationsmanagement, S. 121-140

Scholl, W. (Hrsg.) (2006): Innovation und Information, Göttingen Spath, D./Ganz, W. (2009): Die Zukunft der Dienstleistungswirtschaft: Trends und Chancen heute erkennen, München

von Hippel, E. (1978): A customer active paradigm for industrial product idea generation, in: Research Policy 7 (3), S. 240-266

von Hippel, E. (2005): Democratizing innovation, Cambridge

Zerfass, A./Möslein, K. (Hrsg.) (2009): Kommunikation als Erfolgsfaktor im Innovationsmanagement, Wiesbaden 